\title{
Parametric study and reliability-based evaluation of alternate load path design in reinforced concrete slabs
}

\author{
Didier Droogné, Robby Caspeele and Luc Taerwe \\ Ghent University, Ghent, Belgium \\ Borja Herraiz \\ Dr. Lüchinger + Meyer Bauingenieure AG, Zürich, Switzerland
}

Contact: didier.droogne@ugent.be

\begin{abstract}
In normal design situations, RC slabs are in general designed using small deformation theories while taking into account linear elastic behaviour. However as indicated by previous large structural failures the importance of considering the behaviour of RC slabs at large deformations is as important. Based on multiple experimental studies it is clear that RC slabs can develop alternate load paths and consequently generate a significant strength reserve by membrane action once large deformations occur due to the removal of a load-bearing element. This strength reserve is of major importance as this could result in an important increase of the structural robustness for RC buildings. In this contribution a parametric study with a numerical model is performed to investigate the design possibilities on membrane action in RC slabs. Next the reliability of the developed membrane action and alternate load path is calculated for a reference case which is subjected to the removal of a central support considering the static and pseudo-static behaviour.
\end{abstract}

Keywords: Alternate load paths, membrane action, structural robustness, parametric study, reliability study, $\mathrm{RC}$ slabs

\section{Introduction}

Regarding past large structural failures, such as the collapse at Ronan Point (1968) and the bombing of the Murrah Federal Building (1995), the necessity to design for structural robustness has been underlined and recognized in the engineering community. In general structural robustness can be defined as the property of a structure to survive an extreme event, including man-made and natural hazards, without being damaged to an extent disproportional to the initiating event. Taking into account the initiating event, parts of the building are mostly subjected to some local damage and consequently to large deformations. In case of a robust design this local damage should not result in disproportional or progressive failure in the structural system. One of the strategies to decrease the danger on progressive collapse is the provision of alternate load paths which increase the continuity and structural redundancy within the structure and allow a redistribution of loads to limit the damage extent. Based on experimental and numerical experiments, it is clear that RC slabs may provide significant alternate load paths by the development of membrane action at large deformations. To model this membrane action in simply supported laterally unrestrained slabs, Herraiz [1] developed a numerical model which has been verified by multiple experimental results. In this contribution, the numerical model of Herraiz is extended to perform a parametric study on some 
design parameters. This parametric study enables to investigate the possibilities of the alternate load path design in RC slabs. Next as it is also important to know the variability of the load-bearing resistance of the alternate load path, a reliability study is performed on the resistance of RC slabs when subjected to large deformations due to the removal of a central load-bearing column. The removal of a load-bearing column is chosen to examine the potential for progressive collapse, as this provides a hazard-independent generic local damage which represents a large range of possible events and threats. For the reliability study the static behaviour and pseudo-static behaviour of the $\mathrm{RC}$ slab are taken into account. Considering the pseudo-static behaviour, dynamic effects possibly involved with the initiating event are taken into account.

\section{Membrane action in RC slabs}

Traditionally RC slabs and beams are designed by applying small deformation theories while considering the $\mathrm{RC}$ elements as flexural members which have to carry the applied loads by the development of moment and shear stresses. However when one of the supports of a RC beam or slab is removed due to an exceptional event the RC elements are subjected to large deformations. Due to the larger deformations, in-plane forces or membrane action develop in the elements which initiate alternate load paths and activate some strength reserve. The analytical and experimental research on the strength reserve by membrane action started mid- $20^{\text {th }}$ century focusing firstly on compressive membrane action [2]. Hereby it was noticed that RC slabs could bear significantly more loads than predicted by Johanssens yield-line theory. Later experimental research on restrained slabs subjected to large deflections demonstrated the strength reserve of the tensile membrane action for small to medium scale specimens with a small thickness [3]. For two-way carrying unrestrained slabs subjected to large deformations, tensile membrane action in the center of the slab and compressive membrane action at the edges of the slab was shown to be the most important load carrying mechanism [4].
In general the behavior of restrained and unrestrained slabs can be presented as shown in Figure 1.

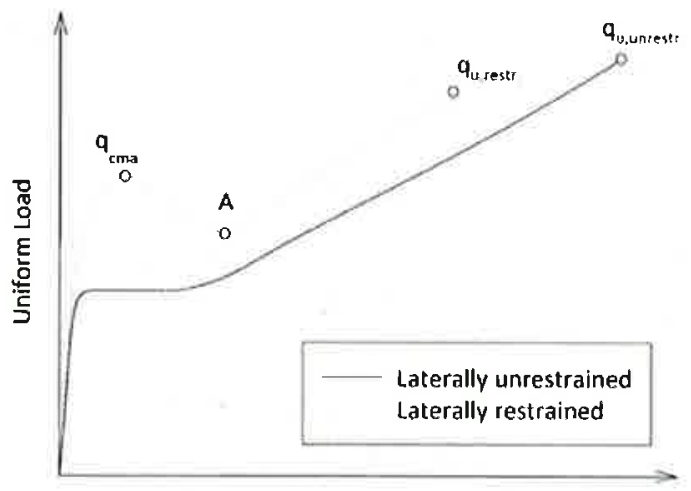

Displacement at the middle of the slab

Figure 1. Approximate load-deflection diagram for. two-way restrained and unrestrained $R C$ slabs

First both types of slabs are characterized by a linear elastic uncracked phase at small loads and displacements which is followed by a plastic phase which has a lower stiffness due to the development of concrete cracks and plastic deformations of the reinforcement. For restrained slabs at small deflections of the slab in-plane, compressive membrane forces are induced as a result of the restrained outward displacements of the slab. The slab tries to move or expand outward but due to the restraints this is not possible and compressive membrane action develops. Next the development of these membrane forces activate a significant beneficial effect as the bending moment $(\mathrm{M})$ and normal force $(\mathrm{N})$ interaction results in a much larger maximal flexural load than normally accounted for by traditional small-displacement theories. At larger displacements the resistance of the slab starts to decrease and some softening is observed in the load-displacement graph due to the gradual reduction of the compressive membrane forces. During this phase the concrete in the compressive zones has reached its maximal strength and starts to crush for larger deformations. Next at point $\mathrm{A}$ in Figure 1, which generally corresponds to a deflection at the center of the slab equal to the effective depth of the slab, the lateral displacements inverse and the slab edges tend to move inwards due to the kinematic relationships. As a consequence the membrane forces change from compression to tension and the edge restraints start to resist inward movement of 
the slab. At this point the tensile membrane phase initiates and the reinforcement starts acting as a tensile net which enables additional load-carrying capacity. If sufficient reinforcement and ductility is available the resistance can increase beyond the maximal load bearing capacity of the compressive membrane phase. Eventually the element fails due to rupture of the reinforcement (Point $q_{u, r e s t r}$ in Figure 1). Note that the softening part will only be observed in a displacement controlled test. In practice under gravity loading, snap-through behavior may be observed.

For unrestrained slabs a similar behavior can be observed, except for the compressive membrane phase as the lateral outward displacement is not restrained. Consequently for small deflections the load-bearing capacity of unrestrained slabs does not exceed the load-bearing capacity predicted by the yield-line theory. On the other hand for larger deflections (generally corresponding to deflections larger than the effective depth of the slab) the central part of the slab starts to develop tensile membrane action while a balancing compressive ring is created at the perimeter of the slab due to the kinematic relationships of the slab segments. Next the tensile membrane action develops further until the slab fails due to concrete crushing or

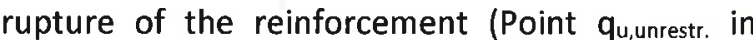
Figure 1), given that sufficient ductility and reinforcement is available.

\section{Calculations}

\subsection{Applied numerical model}

Based on the kinematics of a rigid perfectly-plastic slab model and the equilibrium of the internal forces originating from the corresponding deformations, Herraiz developed a new design approach to calculate the response of flat unrestrained simply supported slabs which are subjected to large deformations [1]. As large deformations are considered, this model includes non-linear effects originating from the material and geometrical non-linearities. To validate the model, the model has been compared with a set of 45 scaled experimental results, originating from different experimental campaigns. This comparative validation suggests that the proposed method generally results in more precise and more reliable results than other approaches found in literature. Therefore in this contribution the model by Herraiz is applied for a parametric and reliability study on the membrane behaviour of full-scale RC slabs.

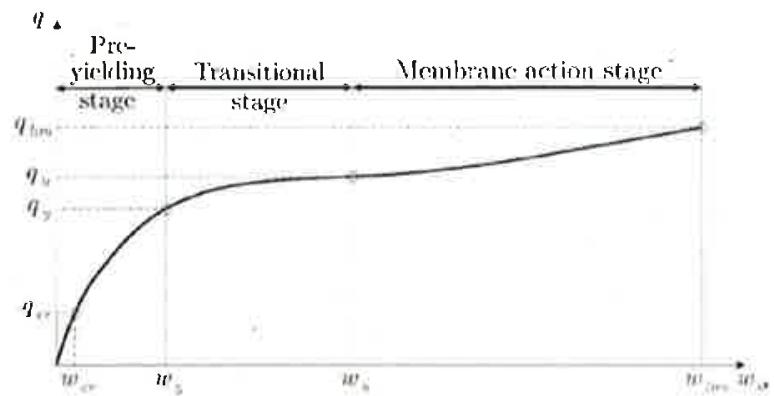

Figure 2. Load-deflection diagram of the applied design method [1]

The developed design approach of the method of Herraiz considers a load-deflection behaviour consisting of three main stages (Figure 2). First the behaviour at small deformations is governed by the linear elastic properties of the slab till the first yielding in the slab occurs ( $q_{y}$ and $w_{y}$ in Figure 2$)$. This stage is called the pre-yielding stage. Next, after first yielding occurs within the slab, the behaviour of the slab is governed by elastic-plastic interactions till the full kinematic model and yield lines develop which can be determined by the classic yield line theory $\left(q_{u}\right.$ and $w_{u}$ in Figure 2$)$. This stage is called the transitional stage as it represents the transition of the slab behaviour from flexural to membrane behaviour. The behaviour of the slab during the final stage or membrane action stage is determined based on the assumed kinematic model which consists of perfect yield lines and rigid slab segments (Figure 3 ). One of the assumptions is that the yield-line collapse pattern of the kinematic model does not change for large deflections. Another important assumption of this design method is the development of an axial crack (Figure 3 ), as been observed in experiments. In this contribution the following material models are used: a parabolic-rectangular stress distribution for the concrete in compression, as defined by the Probabilistic Model Code (PMC) [5] of the Joint Committee on Structural Safety (JCSS) and an elastic-plastic stress-strain relationship for the reinforcement . To determine the ultimate 
resistance of the slab ( $q_{\text {lim }}$ and $w_{\text {lim }}$ in Figure 2) two failure criteria are implemented, namely (1) compressive failure due to concrete crushing and (2) reinforcement rupture at the axial crack. More information on the applied design method can be found in [1].

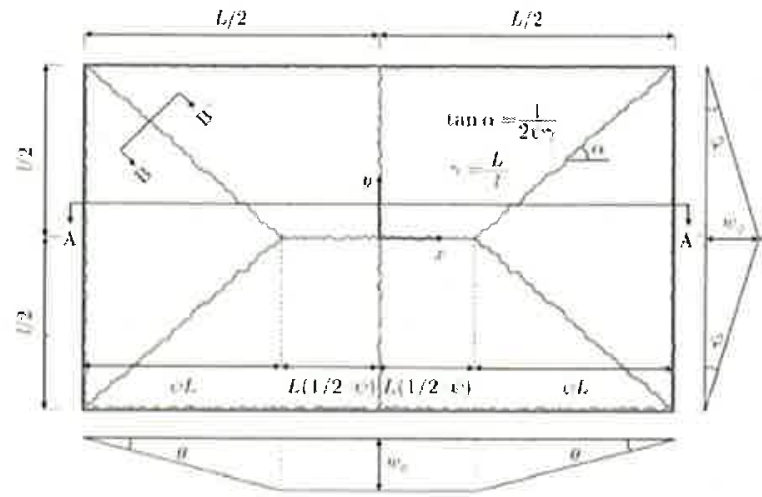

Figure 3. Assumed yield line pattern of the applied design method [1]

\subsection{Reference case}

As a reference case a regular continuous slab of an office building is considered which is designed according to the Eurocodes [6]. In the normal design situation the rectangular slab has a span of 5 and $6 \mathrm{~m}$ in the two orthogonal directions $x$ and $y$ respectively. Next, when considering the removal of a central support in the accidental situation, the spans double to 10 and $12 \mathrm{~m}$ respectively. The membrane action of the slab is investigated under the removal of a central support as this provides a hazard-independent method to assess the potential of progressive collapse. The loads considered in the design of the slab are a service load $Q$ of $3.0 \mathrm{kN} / \mathrm{m}^{2}$ and the imposed self-weight $G$ of the slab and finishing layer (thickness $=60 \mathrm{~mm}$ ). The concrete material is class $\mathrm{C} 30 / 37$ and the reinforcing steel is a BE500S quality with a ductility class B. Taking into account table 7.4N of EN 19921-1 the slenderness ratio $\lambda$ is assessed to be 30 , which results in a total height of $195 \mathrm{~mm}$ for a concrete cover of $30 \mathrm{~mm}$. [6]. Next, calculating the moment coefficients by integrating the differential equations of a clamped slab according to the thin plate bending theory, the necessary reinforcement from a structural viewpoint is calculated. For the latter it is assumed that the admissible steel stresses are limited to $250 \mathrm{MPa}$ to avoid problems with deflections and cracks in the serviceability limit state, and it is assumed that the internal lever arm of the internal forces in the slab is equal to $0.9 d$ with $d$ the effective depth of the slab. Moreover the minimal and maximal reinforcement according to EN 1992-1-1 are taken into account [6].

In Table 1 the design of the reinforcement is summarized. As a simplification for the numerical model no curtailment of the reinforcement layers is considered.

Table 1. Design of Reinforcement

\begin{tabular}{|l|l|l|l|}
\hline $\begin{array}{l}\text { Position } \\
\text { reinforcement }\end{array}$ & Symbol & $\begin{array}{l}\varnothing \\
{[\mathrm{mm}]}\end{array}$ & $\begin{array}{l}\text { Spacing } \\
\text { [mm] }\end{array}$ \\
\hline X-dir. bottom & $A_{s b, x}$ & 8 & 210 \\
\hline X-dir. top & $A_{s t, x}$ & 10 & 225 \\
\hline Y-dir. bottom & $A_{s b, y}$ & 8 & 200 \\
\hline Y-dir. top & $A_{s t, y}$ & 10 & 210 \\
\hline
\end{tabular}

\subsection{Input parameters for the LHS-samples}

To investigate the variability in structural bearing capacity of the reference case the numerical model is combined with Latin-Hypercube Sampling (LHS) [7] and consequently the structural reliability is assessed. LHS allows to limit the number of numerical calculations necessary for a probabilistic assessment to a reasonable amount. Based on the PMC provided by the JCSS a set of eight variables related to the material properties are considered for the LHS procedure (Table 2) [5]. With these eight variables it is possible to implement the material models in a probabilistic way. According to the PMC the stress-strain relationship for the concrete material in compression is given by equations 3-6 [5]. The Young's modulus and tensile strength of the concrete is calculated by equation 7 and 8 respectively.

$$
\begin{aligned}
& \epsilon_{c} \leq \epsilon_{s}: \quad \sigma_{c}=f_{c} \cdot\left[1-\left[1-\frac{\epsilon_{c}}{\epsilon_{s}}\right]^{k}\right] \\
& \epsilon_{c}>\epsilon_{s}: \quad \sigma_{c}=f_{c} \\
& \epsilon_{s}=0.0011 \cdot f_{c}^{1 / 6}
\end{aligned}
$$




$$
\begin{aligned}
& k=\frac{E_{c} \epsilon_{s}}{f_{c}} \\
& \epsilon_{c u}=6 \cdot 10^{-3} f_{c}^{-1 / 6} Y_{4} \\
& f_{c t}=0.3 f_{c}^{2 / 3} Y_{2} \\
& E_{c}=10.5 f_{c}^{1 / 3} Y_{3}
\end{aligned}
$$

with

- $\sigma_{c}$ is the concrete stress [MPa];

- $f_{c}$ is the concrete compressive strength [MPA]

- $\varepsilon_{c}$ is the concrete strain [-];

- $\varepsilon_{S}$ is the concrete limit elastic compr. strain [-]

- $\varepsilon_{c u}$ is the concrete ultimate compr. strain [-];

- $E_{c}$ is the concrete modulus of elasticity [GPa];

- $f_{c t}$ is the concrete tensile strength [MPa];

- $Y_{2}, Y_{3}$ and $Y_{4}$ are model uncertainties reflecting variations due to factors not well accounted for by the concrete compressive strength.

Table 2. Prob. models for material parameters

\begin{tabular}{|l|c|c|c|c|}
\hline Variable & Symb. & Distr. & Mean & COV. \\
\hline Concrete compr. strength & $f_{c}$ & LN & $\begin{array}{c}38.0 \\
\mathrm{MPa}\end{array}$ & 0.10 \\
\hline $\begin{array}{l}\text { Model uncert. tensile } \\
\text { strength }\end{array}$ & $Y_{2}$ & $\mathrm{LN}$ & 1.0 & 0.30 \\
\hline Model uncert. E-modulus & $Y_{3}$ & $\mathrm{LN}$ & 1.0 & 0.15 \\
\hline $\begin{array}{l}\text { Model uncert. ultimate } \\
\text { strain of concrete }\end{array}$ & $Y_{4}$ & $\mathrm{LN}$ & 1.0 & 0.15 \\
\hline $\begin{array}{l}\text { Yield strength } \\
\text { reinforcement }\end{array}$ & $f_{y}$ & $\mathrm{~N}$ & $\begin{array}{c}560 \\
\mathrm{MPa}\end{array}$ & 0.05 \\
\hline $\begin{array}{l}\text { Ratio yield and ultimate } \\
\text { strength of reinforcement }\end{array}$ & $k$ & $\mathrm{LN}$ & 1.23 & 0.05 \\
\hline Young's modulus reinf. & $E_{s}$ & $\mathrm{LN}$ & $\begin{array}{l}205 \\
\mathrm{GPa}\end{array}$ & 0.08 \\
\hline Ultimate Strain reinf. & $\varepsilon_{s u}$ & $\mathrm{~N}$ & $5.7 \%$ & 0.09 \\
\hline
\end{tabular}

For the reinforcing steel a ductility class $B$ according to EC 1992-1-1 is implemented with a characteristic yield strength of $500 \mathrm{MPa}$ and a characteristic ultimate strain of $5 \%$ [6]. In total 100 samples were used for the LHS sample.

\section{Results}

\subsection{Parametric study}

To investigate the influence of some design parameters on the developed membrane action of RC slabs a parametric study was performed. The parameters which are investigated are the concrete compressive strength $f_{c}$, the yield strength of the reinforcement $f_{y}$, the ultimate strain of the reinforcement $\varepsilon_{s u}$, the reinforcement ratio $\rho$ and the aspect ratio $\gamma=L / l$ of the slab. Note that for each parameter study the mean values from Table 2 or reference design parameters from section 4.1 are used for the parameters which are not investigated in the respective parametric study. The parametric study is performed for the slab in the accidental state, i.e. under the removal of the central support, as membrane action is more important to consider in the accidental situation than in the original undamaged situation.

\subsubsection{Material related parameters}

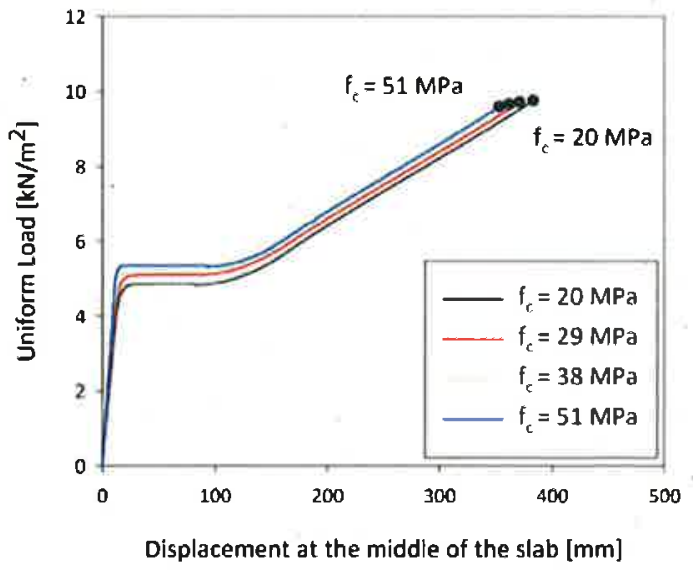

Figure 4. Influence of the concrete compressive strength $f_{c}$

As the RC slabs mostly fail by rupture of the reinforcement in the axial crack, the influence of the concrete strength $f_{c}$ on the ultimate strength of the slab $q_{\text {lim }}$ is negligible (Figure 4). Increasing $f_{c}$ results in a slightly higher yield load $q_{u}$ and a slight decrease in the deformation capacity $w_{1 \text { lim }}$ of the slab. Consequently, increasing $f_{c}$ slightly decreases the beneficial influence of the membrane action 
compared to the yield resistance of the slab, i.e. a decrease of the ratio $q_{l i m} / q_{u}$.

Regarding the reinforcing steel a parametric study on the yield strength $f_{y}$ and on the ultimate strain of the reinforcement $\varepsilon_{s u}$ is performed. Increasing the yield strength results in an increase of the yield resistance and ultimate strength of the slab. Likewise increasing the ultimate strain of the reinforcement results in a significant increase of the ultimate strength of the slab. By increasing the ultimate strain, the slab has a more ductile behaviour. As a consequence for larger $\varepsilon_{s u}$, significant larger deflections can be obtained and the tensile membrane action of the slab can be developed further which results in a higher loadbearing capacity of the slab.

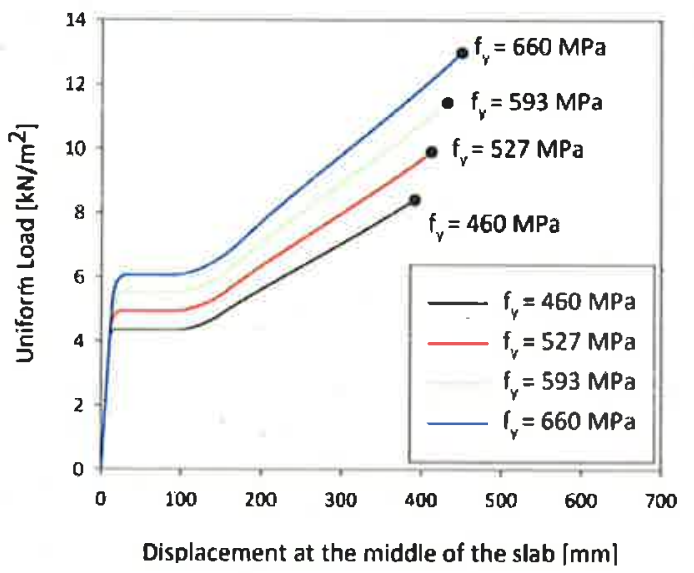

Figure 5. Influence of the reinforcement yield strength $f_{y}$

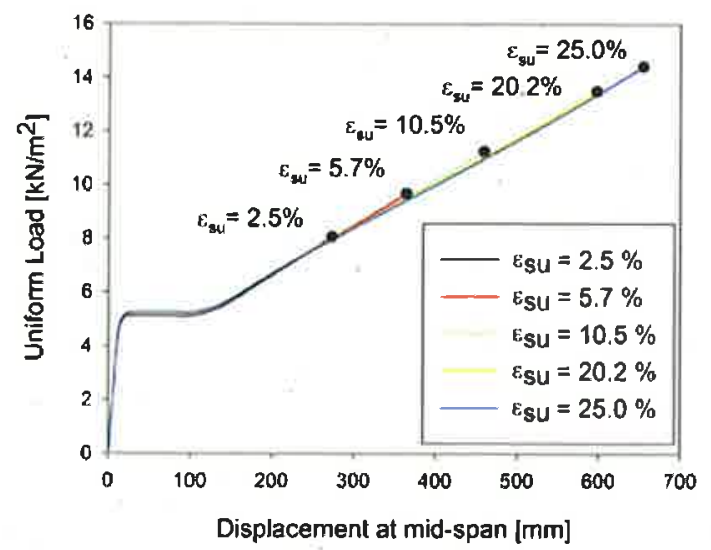

Figure 6. Influence of the reinforcement ultimate strain $\varepsilon_{s u}$

\subsubsection{Geometrical related parameters}

Besides the material properties, two geometrical parameters are investigated. Regarding the aspect ratio $\gamma$, the following can be concluded (Figure 7). The closer the aspect ratio is to 1 the more the membrane action can be developed and the larger the increase in load-bearing capacity by membrane action. The larger the aspect ratio $\gamma$, the more the slab will behave as a one-way carrying slab for which the compressive ring and tensile net can be less developed and less membrane action can be generated. Note that a constant reinforcement ratio $\rho$ of $0.4 \%$ was maintained during this parametric study for the entire slab.

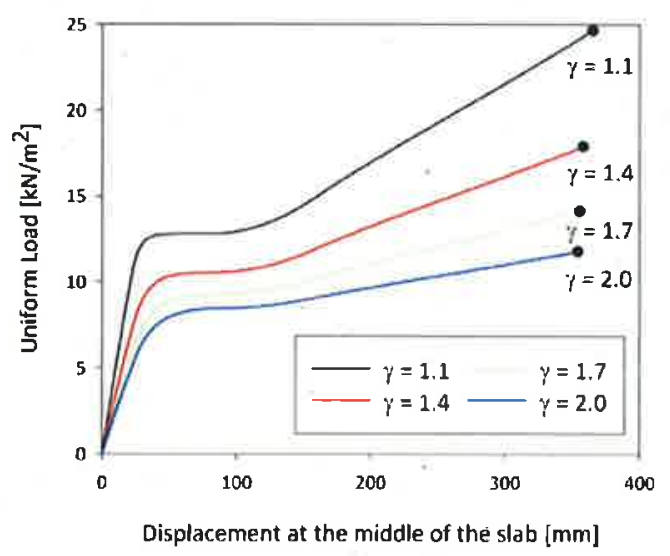

Figure 7. Influence of the aspect ratio $\gamma$ of the slab

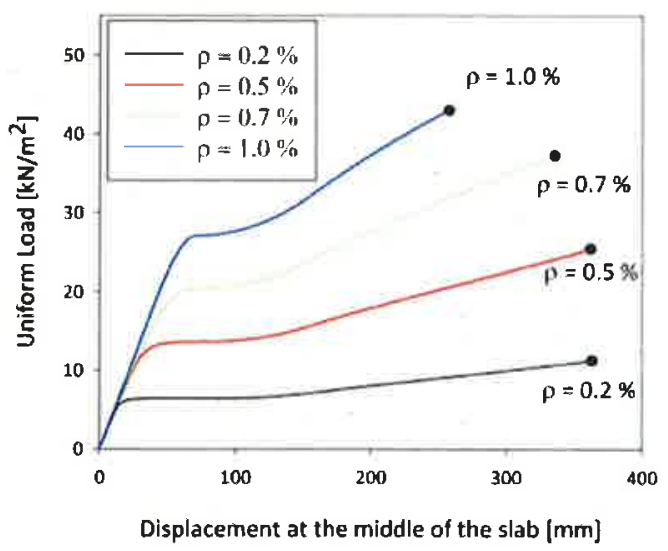

Figure 8. Influence of the reinforcement ratio $\rho$

Increasing the reinforcement ratio results logically in a larger resistance of the slab. As more reinforcement is available for larger reinforcement ratios, the resistance of the tensile net in case of tensile membrane action increases. However the deformation capacity of the slab also decreases for larger reinforcement ratios. The latter can be 
explained by the change in failure mechanism. For small reinforcement ratios, failure of the slab is governed by rupture of the reinforcement in the axial crack whereas for large reinforcement ratios, failure of the slab is governed by crushing of the concrete in the compressive ring at smaller deflections.

\subsection{Reliability study}

Implementing the material parameters of each LHS sample in the numerical model, a set of loaddisplacement diagrams is obtained. Next, for each load-displacement diagram the maximal resistance of the slab $R_{\text {slab }}$ can be determined and a distribution can be fit to the obtained set of slab resistances. Regarding the obtained results a lognormal distribution is chosen to represent the uncertainty related to the resistance. Combining the distribution of the slab resistance with the distributions of the loads, following limit state equation can be used to determine the failure probability of the slab:

$$
g=R_{\text {slab }}-G t-Q
$$

with $t$ being the total thickness of the slab and the finishing layer (255 $\mathrm{mm}$ ).

The applied probabilistic models for the variable service load $Q$ and self-weight $G$ in Table 3 are based on the suggestions of the PMC of JCSS and the recommendations provided by Holicky and Sykora $[5,8]$.

Table 3. Prob. Models for the reliability study

\begin{tabular}{|l|l|l|l|l|}
\hline Variable & Symb. & Distr. & Mean & CoV. \\
\hline $\begin{array}{l}\text { Slab } \\
\text { resistance }\end{array}$ & $R_{\text {slab }}$ & $\mathrm{LN}$ & $\begin{array}{l}11.22 \\
\mathrm{kN} / \mathrm{m}^{2}\end{array}$ & 0.14 \\
\hline $\begin{array}{l}\text { Slab } \\
\text { resistance } \\
\text { Dynamic }\end{array}$ & $R_{\text {slab,dyn }}$ & $\mathrm{LN}$ & $\begin{array}{l}7.23 \\
\mathrm{kN} / \mathrm{m}^{2}\end{array}$ & 0.11 \\
\hline $\begin{array}{l}\text { Self-weight } \\
\text { Service Load }\end{array}$ & $\mathrm{G}$ & $\mathrm{N}$ & $24 \mathrm{kN} / \mathrm{m}^{3}$ & 0.04 \\
\hline & $\mathrm{GU}$ & $\begin{array}{l}0.6 \\
\mathrm{kN} / \mathrm{m}^{2}\end{array}$ & 1.1 \\
\hline
\end{tabular}

Taking the distributions of Table 3 and equation (9) into account a failure probability of $0.2 \%$ or a reliability index $\beta$ of 2.871 is obtained. As a consequence it can be concluded that in case of the removal of a central support, the reliability of the slab is acceptable due to the developed beneficial alternate load path by tensile membrane action.

Note that the calculations above are based on a static approach. Using an energy-based quasi-static approach the dynamic behaviour of the column removal can be considered. The quasi-static approach is based on an energy-balancing method which equals the dissipated internal deformation or strain energy with the energy of the external loads to calculate the dynamic capacity curve of the slab (Figure 9)[9].

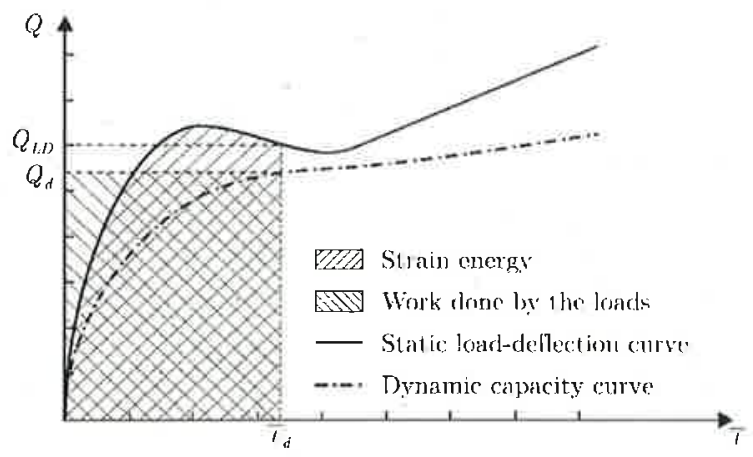

Figure 9. Graphical representation pseudo-static approach [9]

Applying the energy-balancing pseudo-static approach to all obtained load-displacement diagrams, a set of dynamic capacity curves can be obtained. Next the maximal dynamic resistance of each sample can be determined for which a distribution can be fit. As can be seen in Table 3, taking into account the dynamic behaviour of the slabs using the pseudo-static approach results in a significant decrease of the slab resistance. The mean value and COV of the resistance decreases by $36 \%$ and $21 \%$, respectively. Replacing $R_{\text {slab }}$ by $R_{\text {slab,dyn }}$ in equation (9), the failure probability and reliability index of the slab can be determined while taking into account the dynamic behaviour of the removal of the central support. For the latter a failure probability of $28.7 \%$ or a reliability index $\beta$ of 0.563 is obtained, which is a significant increase of the failure probability compared to the static approach. 


\section{Discussion}

Regarding the obtained results following remarks should be made:

- The numerical model is subjected to some assumptions which can have an influence on the obtained results. For instance it is assumed that the yield-line pattern is fixed and does not change at large deflections;

- Moreover the numerical model is only validated for scaled experiments which may behave differently than full-scale elements;

- The previous reliability analyses are related to a specific damage situation in a specific structural configuration. Hence, the established results and failure probabilities should be treated with care and should be considered as indicative only.

\section{Conclusion}

In this contribution the numerical model of Herraiz which enables to model the membrane behaviour of simply supported laterally unrestrained slabs subjected to the removal of a central support is extended to perform a parametric and reliability study to investigate the possibilities of membrane action in RC slabs. Based on the results of the parametric study, it is clear that changing the properties of the reinforcement has a larger influence than changing the concrete compressive strength, as the slabs mainly fail by rupture of the reinforcement. Increasing the amount, the yield strength or ductility of the reinforcement results in a significant increase of the slab capacity. Next it is clear that the tensile membrane net develops more easily for small aspect ratios. Based on the structural reliability calculations, it is clear that in case a central support is removed and the span lengths are doubled, the failure probability remains reasonably low due to the formation of the alternate load path by membrane action. However taking into account the dynamic behaviour of the considered accidental situation, a significant increase of the failure probability is observed. As a consequence taking into account the dynamic behaviour is of major importance.

\section{Acknowledgements}

The authors wish to thank Ghent University for the financial support from the Special Research Fund (BOF) on the research project "Risk and reliabilitybased evaluation and development of design guidelines for structural robustness measures in constructions taking into account membrane action".

\section{References}

[1] Herraiz B. and Vogel T. Novel design approach for the analysis of laterally unrestrained reinforced concrete slabs considering membrane action. Eng. Struct. 2016; 123: 313-329.

[2] Park R. Tensile membrane behaviour of uniformly loaded rectangular reinforced concrete slabs with fully restrained edges. Mag. Concr. Res. 1964; 16(46): 39-44.

[3] Foster. S. et al. Experimental behaviour of concrete floor slabs at large displacements. Eng. Struct. 2004; 26(9): 1231-1247.

[4] Bailey C. G. Membrane action of unrestrained lightly reinforced concrete slabs at large displacements. Eng. Struct. 2001; 23(5): 470483.

[5] JCSS. Probabilistic Model Code. 2001.

[6] CEN. EN 1992-1-1: Design of concrete structures - Part 1-1: General rules and rules for buildings. 2005

[7] Olsson A., Sandberg G. and Dahlblom O. On Latin Hypercube Sampling for Structural Reliability Analysis. Struct. Safety. 2003; 25(1): 47-68.

[8] Holicky M. and Sykora M. Stochastic models in analysis of structural reliability. Proc. Int. Symp. on stochastic models in reliability engineering, Life Sciences and Operations Management. Israel. 2010.

[9] Herraiz B., 'Russel J. and Vogel T. Energy-based method for sudden column failure scenarios: theoretical, numerical and experimental analysis. Proc. of IABSE workshop Helsinki. 2015: 70-77. 